\title{
SS-LIFTING MODULES AND RINGS
}

\author{
FIGEN ERYILMAZ
}

Received 22 February, 2020

\begin{abstract}
A module $M$ is called ss-lifting if for every submodule $A$ of $M$, there is a decomposition $M=M_{1} \oplus M_{2}$ such that $M_{1} \leq A$ and $A \cap M_{2} \subseteq \operatorname{Soc}_{s}(M)$, where $\operatorname{Soc}_{s}(M)=\operatorname{Soc}(M) \cap \operatorname{Rad}(M)$. In this paper, we provide the basic properties of $s s$-lifting modules. It is shown that: (1) a module $M$ is $s s$-lifting iff it is amply ss-supplemented and its $s s$-supplement submodules are direct summand; (2) for a ring $R,{ }_{R} R$ is $s s$-lifting if and only if it is $s s$-supplemented iff it is semiperfect and its radical is semisimple; (3) a ring $R$ is a left and right artinian serial ring and $\operatorname{Rad}(R) \subseteq \operatorname{Soc}\left({ }_{R} R\right)$ iff every left $R$-module is $s s$-lifting. We also study on factor modules of ss-lifting modules.
\end{abstract}

2010 Mathematics Subject Classification: 16D10; 16D60

Keywords: semisimple module, ss-supplemented module, strongly local module.

\section{INTRODUCTION}

In this study $R$ is used to show a ring which is associative and has an identity. All mentioned modules will be unital left $R$-module. Let $M$ be an $R$-module. The notation $A \leq M$ means that $A$ is a submodule of $M$. A proper submodule $A$ of $M$ is called small in $M$ and showed by $A \ll M$ whenever $A+C \neq M$ for all proper submodule $C$ of $M$. A module $M$ is called hollow if every submodule of $M$ is small in $M$. By $\operatorname{Rad}(M)$, namely radical, we will denote the sum of all small submodules of $M$. Equivalently, $\operatorname{Rad}(M)$ is the intersection of all maximal submodules of $M$. A hollow module $M$ with maximal radical is local. As a dual notion of a small submodule, a submodule $E \subseteq M$ is called essential in $M$, denoted by $E \unlhd M$, if $E \cap K \neq 0$ for every nonzero submodule $K$ of $M$. The socle of $M$ which is the sum of all simple submodules of $M$ is denoted by $\operatorname{Soc}(M)$. It is well known that $\operatorname{Soc}(M)$ is the intersection of all essential submodules of $M$. The relation between radical and socle of a module $M$ is not determined. In [8], the sum of all simple submodules of the module $M$ that is small is denoted by $\operatorname{Soc}_{s}(M)$. It is shown in [4, Lemma 2] that $\operatorname{Soc}_{s}(M)=\operatorname{Soc}(M) \cap \operatorname{Rad}(M)$.

A module $M$ is called extending if every submodule of $M$ is essential in a direct summand of $M$ [3]. Dually, a module $M$ is lifting if for every submodule $A$ of $M$ lies over a direct summand, that is, there is a decomposition $M=M_{1} \oplus M_{2}$ such that $M_{1} \leq A, A \cap M_{2} \ll M_{2}$. A characterization of lifting modules is given with help of 
supplemented modules in [3]. Here a module $M$ is supplemented if every submodule $A$ of $M$ has a supplement $B$ in $M$, that is, $M=A+B$ and $A \cap \ll B$. $M$ is called amply supplemented if whenever $M=A+B, B$ contains a supplement of $A$ in $M$. Clearly, direct summands are supplements. By [7], $M$ is lifting if and only if $M$ is amply supplemented and every supplement submodule of $M$ is a direct summand of it.

Since $\operatorname{Soc}_{s}(X)=\operatorname{Soc}(X) \cap \operatorname{Rad}(X) \ll X$ for any module $X$, the authors call a submodule $V$ of a module $M$ ss-supplement of a submodule $U$ in $M$ if $M=U+V$ and $U \cap V \subseteq \operatorname{Soc}_{s}(V)$ (see [4]). It is shown in [4, Lemma 3] that a submodule $V$ of $M$ is ss-supplement of some submodule $U$ in $M$ if and only if $V$ is a supplement of $U$ in $M$ and $U \cap V$ is semisimple. Following [4], a module $M$ is said to be ss-supplemented if every submodule $A$ of $M$ has an ss-supplement $B$ in $M$, and it is called amply ss-supplemented if whenever $M=A+B, B$ contains an ss-supplement of $A$ in $M$. Clearly, the class of ss-supplemented modules is between the class of semisimple modules and the class of supplemented modules. The basic properties and characterizations of $s s$-supplemented modules are given in the same paper.

Considering all of these definitions, we can define $s s$-lifting modules. A module $M$ is called ss-lifting if for every submodule $A$ of $M$, there is a decomposition $M=M_{1} \oplus M_{2}$ such that $M_{1} \leq A, A \cap M_{2} \ll M$ and $A \cap M_{2}$ is semisimple. In this paper, some fundamental properties of $s s$-lifting modules will be examined. It is proved that a module $M$ is $s s$-lifting module if and only if it is amply $s s$-supplemented and every ss-supplement submodule of $M$ is direct summand. It is shown that every $\pi$-projective and $s s$-supplemented module is $s s$-lifting. It is proved that for a ring $R$, ${ }_{R} R$ is $s s$-lifting if and only if $R$ is semiperfect and its radical is semisimple. Moreover, it is shown that $R$ is a left and right artinian serial ring and $\operatorname{Rad}(R) \subseteq \operatorname{Soc}\left({ }_{R} R\right)$ if and only if every left $R$-module is $s s$-lifting. Nevertheless, it is proved that any factor module generated by submodule of a weakly distributive module is $s s$-lifting.

\section{SS-LifTing MOduleS}

In this section, we examine the basic properties of $s s$-lifting modules. In particular, we give characterizations of some ring classes via ss-lifting modules. Let us begin with the following definition.

Definition 1. Let $M$ be a module. $M$ is called ss-lifting if, for every submodule $U$ of $M, M$ has a decomposition $M=U^{\prime} \oplus V$ such that $U^{\prime} \subseteq U$ and $U \cap V \subseteq \operatorname{Soc}_{s}(V)$.

It can be seen that a module $M$ is $s s$-lifting if and only if, for every submodule $U$ of $M, M$ has a decomposition $M=U^{\prime} \oplus V$ such that $U^{\prime} \subseteq U$ and $U \cap V \subseteq \operatorname{Soc}_{s}(M)$. Note that we shall freely use this fact in this paper. It is clear that every $s s$-lifting module is lifting. The following example shows that in general a lifting module need not be $s s$-lifting.

Example 1. Let $R$ be a local Dedekind domain and $K$ be the quotient field of $R$. Put $M={ }_{R} K$. Then $M$ is hollow and so it is lifting. Since $R$ is a commutative domain and 
$M$ is an injective module, it follows that $M=\operatorname{Rad}(M)$ and $\operatorname{Soc}(M)=0$. Therefore $\operatorname{Soc}_{s}(M)=\operatorname{Soc}(M) \cap \operatorname{Rad}(M)=0$. So $M$ is not ss-lifting.

Lemma 1. Let $M$ be an ss-lifting module. Then $M$ is amply ss-supplemented.

Proof. Let $U$ be any submodule of $M$. By the hypothesis, there are a submodule $V$ of $M$ and $U^{\prime} \leq U$ such that $M=U^{\prime} \oplus V$ and $U \cap V \subseteq \operatorname{Soc}_{s}(V)$. Therefore $M=U+V$. It means that $V$ is an $s s$-supplement of $U$ in $M$ and so $M$ is ss-supplemented. It follows from [4, Proposition 26] that $U^{\prime}$ is $s s$-supplemented as a direct summand of $M$. Now, by modularity law, we can write $U=U \cap M=U \cap\left(U^{\prime} \oplus V\right)=$ $=U^{\prime} \oplus(U \cap V)$ and then $U$ is ss-supplemented by [4, Corollary 24] since $U \cap V$ is semisimple. Hence $M$ is amply $s s$-supplemented according to [4, Proposition 33].

In [4], a module $M$ is said to be strongly local if $M$ is local and its radical is semisimple. Using Lemma 1, we have the next result.

Corollary 1. For the non-zero hollow module $M$, the following are equivalent:

(1) $M$ is strongly local.

(2) $M$ is ss-lifting.

(3) $M$ is amply ss-supplemented.

Proof. (1) $\Rightarrow(2)$ Let $U$ be a proper submodule of $M$. Since $M$ is strongly local, we have $U \subseteq \operatorname{Rad}(M) \subseteq \operatorname{Soc}(M)$ and then $U$ is semisimple. Now, if $U^{\prime}=0$ and $V=M$ are taken, we obtain that $M=U^{\prime} \oplus M, U^{\prime} \leq U$ and $U \cap M=U$ is semisimple. Thus $M$ is $s s$-lifting.

(2) $\Rightarrow(3)$ It is clear that by Lemma 1 .

(3) $\Rightarrow(1)$ By [4, Proposition 15].

Observe from Corollary 1 that the local $\mathbb{Z}$-module $\mathbb{Z}_{8}$ is lifting which is not ss-lifting.

Lemma 2. Let $M$ be a module and $A \leq M$. The following conditions are equivalent:

(1) There is a direct summand $X$ of $M$ such that $X \leq A$ and $\frac{A}{X} \subseteq \operatorname{Soc}_{S}\left(\frac{M}{X}\right)$.

(2) There are a direct summand $X$ of $M$ and a submodule $Y$ of $M$ such that $X \leq A$, $A=X+Y$ and $Y \subseteq \operatorname{Soc}_{s}(M)$.

(3) There is a decomposition $M=X \oplus X^{\prime}$ with $X \subseteq A$ and $X^{\prime} \cap A \subseteq \operatorname{Soc}_{s}(M)$.

(4) A has an ss-supplement $X^{\prime}$ in $M$ such that $X^{\prime} \cap A$ is a direct summand in $A$.

(5) There is a homomorphism $e: M \longrightarrow M$ with $e^{2}=e$ such that $e(M) \leq A$ and $(1-e)(A) \subseteq \operatorname{Soc}_{s}(1-e)(M)$.

Proof. (1) $\Rightarrow(2)$ Since $X$ is a direct summand of $M$, there exists a submodule $X^{\prime}$ of $M$ with $M=X \oplus X^{\prime}$. If both sides of the equality are taken with $A$, we get that $A=X+\left(X^{\prime} \cap A\right)$. Since $\frac{A}{X} \ll \frac{M}{X}$ and $\frac{A}{X}$ is semisimple, $\Psi\left(\frac{A}{X}\right)=X^{\prime} \cap A \ll X$ and $X^{\prime} \cap A$ is semisimple where $\Psi: M \rightarrow X$ is the canonical projection. 
(2) $\Rightarrow$ (3) By the hypothesis, we can write $M=X \oplus X^{\prime}$ for some submodule $X^{\prime}$ of $M$. Then $X^{\prime}$ is a ss-supplement of $X$ in $M$ and so a $s s$-supplement of $A=X+Y$ in $M$ by [4, Lemma 22]. Therefore $X^{\prime} \cap A \subseteq \operatorname{Soc}_{s}\left(X^{\prime}\right)$.

(3) $\Rightarrow$ (4) If we take an intersection the equality $M=X \oplus X^{\prime}$ with $A$, we can write $A=X \oplus\left(X^{\prime} \cap A\right)$. Hence $X^{\prime}$ is a ss-supplement of $A$ in $M$.

(4) $\Rightarrow(5)$ From the hypothesis, we have $M=A+X^{\prime}, X^{\prime} \cap A \subseteq \operatorname{Soc}_{s}\left(X^{\prime}\right)$ and $A=\left(X^{\prime} \cap A\right) \oplus X$ for some $X \subseteq A$. Then $M=A+X^{\prime}=\left(X^{\prime} \cap A\right)+X+X^{\prime}=X+X^{\prime}$ and $\left(X^{\prime} \cap A\right) \cap X=0$ and $M=X+X^{\prime}$. Let $e: M \rightarrow M$ be the projection such that $e(m)=x, m=x+x^{\prime}, x \in X, x^{\prime} \in X^{\prime}$. Then $e(M) \subseteq X \subseteq U$. Since $(1-e)(M)=X^{\prime}$, we get that $(1-e)(A)=X^{\prime} \cap A \ll X^{\prime}=(1-e)(M)$ and $X^{\prime} \cap A$ is semisimple.

(5) $\Rightarrow(1)$ Let $X=e(M)$. Since $e$ is an idempotent, we have $M=e(M) \oplus(1-e)(M)$. Then $M=X \oplus(1-e)(M)$ with $X \subseteq A$. We will consider the isomorphism $\Phi: \frac{M}{X} \rightarrow(1-e)(M)$. From here, $\Phi\left(\frac{A}{X}\right)=(1-e)(A) \ll(1-e)(M)=\Phi\left(\frac{M}{X}\right)$. Since $\Phi^{-1}$ is an isomorphism, we can get $\frac{A}{X} \ll \frac{M}{X}$ and $\Phi^{-1}((1-e)(A))=\frac{A}{X}$ is semisimple. Therefore $\frac{A}{X} \subseteq \operatorname{Soc}_{s}\left(\frac{M}{X}\right)$.

Note that every direct summand of a module is an $s s$-supplement submodule of the module and ss-supplement submodules are supplement.

Theorem 1. For a module $M$, the following conditions are equivalent:

(1) $M$ is ss-lifting.

(2) Every submodule $A$ of $M$ can be written as $A=N \oplus S$ with $N$ is a direct summand of $M$ and $S \subseteq \operatorname{Soc}_{s}(M)$.

(3) $M$ is an amply ss-supplemented module and every ss-supplement submodule of $M$ is a direct summand.

Proof. (1) $\Leftrightarrow(2)$ By Lemma 2.

$(1) \Rightarrow(3)$ It follows from Lemma 1 that $M$ is an amply ss-supplemented module. Since every supplement submodule of a lifting module is a direct summand of the module, it follows from (1) that every every ss-supplement in $M$ is a direct summand.

(3) $\Rightarrow(1)$ Let $A$ be a submodule of $M$. By the hypothesis, $A$ has an $s s$-supplement $X$ and $X$ has an ss-supplement $Y$ such that $Y \leq A$ and $Y$ is a direct summand of $M$. Then there exists a submodule $T$ of $M$ with $M=Y \oplus T$. Hence we get that $A=Y \oplus(A \cap T)$ and $A=Y+(A \cap X)$. If we consider the projection $\pi: Y \oplus T \rightarrow T$, we can obtain that $\pi(A)=\pi(Y+(A \cap X))=A \cap T$. In this way, we say that there is a decomposition $M=Y \oplus T$ such that $Y \leq A, A \cap T \ll M$ and $A \cap T \subseteq \operatorname{Soc}_{s}(M)$ and so $M$ is $s s$-lifting.

Theorem 2. Let $M$ be a $\pi$-projective and ss-supplemented module. Then $M$ is ss-lifting.

Proof. By Proposition 37 of [4], $M$ is amply ss-supplemented. Since $M$ is ss-supplemented, there exists a submodule $V$ of $M$ such that $M=U+V$ and $U \cap V \subseteq \operatorname{Soc}_{s}(V)$. On the other side, there exists a submodule $U$ of $M$ such that 
$M=U^{\prime}+V, U^{\prime} \subseteq U$ and $U^{\prime} \cap V \subseteq \operatorname{Soc}_{s}\left(U^{\prime}\right)$ because $M$ is amply ss-supplemented. Hence $U^{\prime}$ and $V$ are mutual ss-supplements. By 41.14 (2) in [7], we can write $U^{\prime} \cap V=0$. It means that $M=U^{\prime} \oplus V$. Thus $M$ is ss-lifting.

Theorem 3. Let $M$ be an ss-lifting module. Then every direct summand of $M$ is ss-lifting.

Proof. Let $X$ be a direct summand of $M$ with $M=X \oplus X^{\prime}$ for some submodule $X^{\prime}$ of $M$ and $U \leq X$. Since $M$ is $s s$-lifting, there there exists a submodule $V$ of $M$ such that $M=U+V, U \cap V \subseteq \operatorname{Soc}_{s}(V)$. Then we can write $X=U^{\prime} \oplus(X \cap V)$ and $U \cap(X \cap V)=(U \cap X) \cap V=U \cap V$ is semisimple. It follows from $U \cap V \ll M$ and $U \cap V \ll X$ because $X$ is a direct summand of $M$. Hence $X=U^{\prime} \oplus(X \cap V)$ implies that $U \cap V \ll X \cap V$. Thus $X$ is ss-lifting.

Now, we will give necessary conditions for any lifting module to be $s s$-lifting.

Theorem 4. Let $M$ be a module with small radical. Then the following statements are equivalent:

(1) $M$ is ss-lifting.

(2) $M$ is lifting and $\operatorname{Rad}(M) \subseteq \operatorname{Soc}(M)$.

Proof. (1) $\Rightarrow(2)$ Since $\operatorname{Rad}(M)$ is a small submodule of $M$ and $M$ is ss-lifting, $M$ is an $s s$-supplement of $\operatorname{Rad}(M)$ in $M$ and so $\operatorname{Rad}(M) \cap M=\operatorname{Rad}(M)$ is semisimple.

(2) $\Rightarrow(1)$ Let $U \leq M$. Since $M$ is lifting, there is a decomposition for a submodule $V$ of $M, M=U^{\prime} \oplus V, U^{\prime} \leq U$ and $U \cap V \ll V$. It follows that $U \cap V \subseteq \operatorname{Rad}(V) \subseteq$ $\subseteq \operatorname{Rad}(M)$ is semisimple. Thus $M$ is $s s$-lifting.

Since a projective supplemented module has small radical, we have the following fact as a result of Theorem 4 .

Corollary 2. Let $M$ be a projective module. Then $M$ is ss-lifting if and only if it is lifting and its radical is semisimple.

Recall from [7, 43.9] that a ring whose all left modules are supplemented is left perfect. It follows from [7, 43.9] that a ring $R$ is left perfect if and only if $R$ is semilocal and $\operatorname{Rad}(R)$ is right t-nilpotent if and only if every left $R$-module has a projective cover, that is, for any left $R$-module $M$, there exist a projective module $P$ and an epimorphism $f: P \longrightarrow M$ with small kernel. $R$ is called semiperfect if every finitely generated left (or right) $R$-module is supplemented. Now we give a characterization of semiperfect (left perfect) rings.

Lemma 3. Let $R$ be an arbitrary ring. Then ${ }_{R} R$ is ss-lifting if and only if $R$ is semiperfect and $\operatorname{Rad}(R) \subseteq \operatorname{Soc}\left({ }_{R} R\right)$.

Proof. By Theorem 4. 
Theorem 5. The following statements are equivalent for a ring $R$.

(1) ${ }_{R} R$ is ss-lifting.

(2) ${ }_{R} R$ is ss-supplemented.

(3) Every left R-module is ss-supplemented.

(4) $R$ is semiperfect and $\operatorname{Rad}(R) \subseteq \operatorname{Soc}\left({ }_{R} R\right)$.

Proof. (1) $\Rightarrow(2)$ It is clear.

(2) $\Rightarrow(3) \Rightarrow$ (4) It follows from [4, Theorem 41].

(4) $\Rightarrow$ (1) By Theorem 4.

Now we characterize the rings with the property that every left module is $s s$-lifting. Firstly, we need following lemma.

Lemma 4. Let $M$ be a lifting module and $\operatorname{Rad}(M) \subseteq \operatorname{Soc}(M)$.Then $M$ is ss-lifting.

Proof. The proof is clear.

Theorem 6. The following statements are equivalent:

(1) $R$ is a left and right artinian serial ring and $\operatorname{Rad}(R) \subseteq \operatorname{Soc}\left({ }_{R} R\right)$.

(2) Every left $R$-module is ss-lifting.

Proof. (1) $\Rightarrow(2)$ By the hypothesis and Lemma 3, it is clear that $\operatorname{Rad}(R) \subseteq \operatorname{Soc}\left({ }_{R} R\right)$. On the other side, if every left $R$-module is semisimple lifting, then every left $R$-module is lifting by $[2,29.10]$.

$(2) \Rightarrow(1)$ Since $\operatorname{Rad}(R) \subseteq \operatorname{Soc}\left({ }_{R} R\right)$, we have $\operatorname{Rad}(R)^{2}=0$ by [7, 21.12 (4)]. Moreover, we say that every left $R$-module is lifting by [2, 29.10]. We can write $\operatorname{Rad}(M)=\operatorname{Rad}(R) M \subseteq \operatorname{Soc}\left({ }_{R} R\right) M \subseteq \operatorname{Soc}(M)$ because $R$ is an artinian ring. Therefore $M$ is $s s$-lifting by previous Lemma.

Example 2. Consider the local ring $R=\mathbb{Z}_{4}$ is left and right artinian serial ring and $\operatorname{Rad}(R)=\{0,2\}=\operatorname{Soc}\left({ }_{R} R\right)$ and so every left $R$-module is $s s$-lifting by Theorem 6 .

Theorem 7. Let $M$ be a ss-lifting module. If $\frac{K+X}{X}$ is a direct summand of $\frac{M}{X}$ for every direct summand $K$ of $M$, then $\frac{M}{X}$ is ss-lifting.

Proof. Let $\frac{A}{X} \leq \frac{M}{X}$. Since $M$ is ss-lifting, there exists a direct summand $K$ of $M$ with $K \leq A$ and $\frac{A}{K} \subseteq \operatorname{Soc}_{s}\left(\frac{M}{K}\right)$ by Lemma 2. It is clear that $\frac{K+X}{X} \leq \frac{A}{X}$. If we say $\frac{A}{K+X} \subseteq \operatorname{Soc}_{s}\left(\frac{M}{K+X}\right)$, the proof is completed. Since $\frac{\left(\frac{M}{K}\right)}{\left(\frac{K+X}{K}\right)} \cong \frac{M}{K+X}$, we get that $\frac{A}{K+X} \subseteq \operatorname{Soc}_{s}\left(\frac{M}{K+X}\right)$. Therefore, $\frac{M}{X}$ is a ss-lifting module by Lemma 2 .

Recall from [2] that a submodule $U$ of $M$ is called fully invariant if $f(U)$ is contained in $U$ for every $R$-endomorphism $f$ of $M$. Recall from [2] that a module $M$ is called $d u o$ if every submodule of $M$ is fully invariant in $M$.

Theorem 8. Let $M$ be a ss-lifting module and $X$ be a fully invariant submodule of M. Then $\frac{M}{X}$ is ss-lifting. 
Proof. Suppose that $M=K \oplus L$. Then $e(M)=K$ and $(1-e)(M)=L$ for some $e \in \operatorname{End}(M)$. Since $X$ is fully invariant, $e(X)=X \cap K$ and $(1-e)(X)=X \cap L$. From here, $X=e(X) \oplus(1-e)(X)=(X \cap K) \oplus(X \cap L)$ and we can write $\frac{K+X}{X}=$ $\frac{K+[(X \cap K) \oplus(X \cap L)]}{X}=\frac{K \oplus(X \cap L)}{X}$ and $\frac{L+X}{X}=\frac{L+[(X \cap K) \oplus(X \cap L)]}{X}=\frac{L \oplus(X \cap K)}{X}$. Hence $M=$

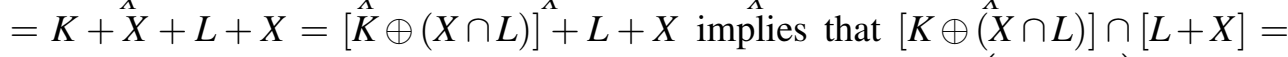
$[K \oplus(X \cap L)] \cap[L+(X \cap K)]=(X \cap K) \oplus(X \cap L)=X$ and $\frac{M}{X}=\left(\frac{K \oplus(X \cap L)}{X}\right) \oplus\left(\frac{L+X}{X}\right)$. Thus $\frac{M}{X}$ is $s s$-lifting by the previous theorem.

Recall from [1] that a submodule $U$ is called a weak distributive of $M$ if $U=(U \cap X)+(U \cap Y)$ for all submodules $X, Y \leq M$ such that $M=X+Y$. A module $M$ is said to be weakly distributive if every submodule of $M$ is a weak distributive submodule of $M$.

Theorem 9. Let $M$ be a weakly distributive module and $X \leq M$. Then $\frac{M}{X}$ is ss-lifting.

Proof. Let $M=K \oplus L$. Then we have $\frac{M}{X}=\left(\frac{K+X}{X}\right)+\left(\frac{L+X}{X}\right)$ and $X=X+K \cap L=$ $(X+K) \cap(X+L)$. Thus $\frac{M}{X}=\left(\frac{K+X}{X}\right) \oplus\left(\frac{L+X}{X}\right)$ and so $\frac{M}{X}$ is $s s$-lifting by Theorem 7 .

Theorem 10. Let $M=M_{1} \oplus M_{2}$ be a duo module. If $M_{1}$ and $M_{2}$ are ss-lifting modules, then $M$ is ss-lifting.

Proof. Suppose that $L$ be a submodule of $M$. We can write $L=\bigoplus_{i=1}^{2}\left(L \cap M_{i}\right)$ by Lemma 2.1 of [2]. For each $i \in\{1,2\}$, there exists a direct summand $D_{i}$ of $M_{i}$ such that $M_{i}=D_{i} \oplus D_{i}^{\prime}$ with $D_{i} \leq L \cap M_{i}$ and $L \cap D_{i}^{\prime} \subseteq \operatorname{Soc}_{s}\left(D_{i}^{\prime}\right)$. From here

$$
M=M_{1} \oplus M_{2}=\left(D_{1} \oplus D_{1}^{\prime}\right) \oplus\left(D_{2} \oplus D_{2}^{\prime}\right)=\left(D_{1} \oplus D_{2}\right) \oplus\left(D_{1}^{\prime} \oplus D_{2}^{\prime}\right) .
$$

It is clear that $D_{1} \oplus D_{2} \leq L$. Since $L \cap D_{i}^{\prime} \subseteq \operatorname{Soc}_{s}\left(D_{i}^{\prime}\right), L \cap\left(D_{1}^{\prime} \oplus D_{2}^{\prime}\right) \subseteq \operatorname{Soc}_{s}\left(D_{1}^{\prime} \oplus D_{2}^{\prime}\right)$. Therefore $M$ is $s s$-lifting.

Lemma 5 (see [5, Lemma 5]). The following statements are equivalent for a module $M=M_{1} \oplus M_{2}$.

(i) $M_{2}$ is $M_{1}$-projective.

(ii) For each submodule $N$ of $M$ with $M=M_{1}+N$ there exists a submodule $N^{\prime}$ of $N$ such that $M=M_{1} \oplus N^{\prime}$.

Theorem 11. Let the module $M=M_{1} \oplus M_{2}$ with $M_{1}$ and $M_{2}$ are relatively projective modules. If $M_{1}$ is semisimple and $M_{2}$ is ss-lifting, then $M$ is ss-lifting.

Proof. Suppose that $K$ be a non-zero submodule of $M$.

Case 1: Assume that $T=M_{1} \cap\left(K+M_{2}\right) \neq 0$. Since $M_{1}$ is semisimple, we can write $M_{1}=T \oplus T_{1}$ for some submodule $T_{1}$ of $M_{1}$ and so $M=T \oplus T_{1} \oplus M_{2}=$ $=\left[\left(M_{1} \cap\left(K+M_{2}\right)\right)\right] \oplus T_{1} \oplus M_{2}=K \oplus\left(M_{2} \oplus T_{1}\right)$. Using Prop. 4.31, Prop. 4.32 and 
Prop. 4.33 in [6], we can say that $T$ is $M_{2} \oplus T_{1}$-projective. By 41.14 in [7], there exists a submodule $K_{1}$ of $K$ such that $M=K_{1} \oplus\left(M_{2} \oplus T_{1}\right)$. Let $A$ be any submodule of $M_{2}$ and $K \cap\left(M_{2} \oplus T_{1}\right) \neq 0$. Since $K \cap\left(A+T_{1}\right) \leq A \cap\left(K+T_{1}\right)+T_{1} \cap(K+A)$ and $T_{1} \cap(K+A)=0$, then $K \cap\left(A+T_{1}\right) \leq A \cap\left(K+T_{1}\right)$. Similarly, $A \cap\left(K+T_{1}\right) \leq$ $K \cap\left(A+T_{1}\right)$. Hence $A \cap\left(K+T_{1}\right)=K \cap\left(A+T_{1}\right)$. Moreover, if we consider $M_{2}$ is ss-lifting, then there exists a submodule $X_{1}$ of $M_{2} \cap\left(K+T_{1}\right)=K \cap\left(M_{2}+T_{1}\right)$ such that $M_{2}=X_{1} \oplus X_{2}$ and $X_{2} \cap\left(K+T_{1}\right) \subseteq \operatorname{Soc}_{s}\left(X_{2}\right)$ for some submodule $X_{2}$ of $M_{2}$. Therefore $M=\left(K_{1} \oplus X_{1}\right) \oplus\left(X_{2} \oplus T_{1}\right), \quad K_{1} \oplus T_{1} \leq K \quad$ and $K \cap\left(X_{2} \oplus T_{1}\right)=$ $=X_{2} \cap\left(K+T_{1}\right) \subseteq \operatorname{Soc}_{s}\left(X_{2} \oplus T_{1}\right)$.

Case 2: Assume that $T=M_{1} \cap\left(K+M_{2}\right)=0$. From here $T$ is a submodule of $M_{2}$. Since $M_{2}$ is ss-lifting, there exists a submodule $Y_{1}$ of $K$ such that $M_{2}=Y_{1} \oplus Y_{2}$, $K \cap Y_{2} \subseteq \operatorname{Soc}_{s}\left(Y_{2}\right)$ for some submodule $Y_{2}$ of $M_{2}$. Thus $M=M_{1} \oplus M_{2}=$ $=M_{1} \oplus\left(Y_{1} \oplus Y_{2}\right)=Y_{1} \oplus\left(M_{1} \oplus Y_{2}\right)$ and $K \cap\left(M_{1} \oplus Y_{2}\right)=K \cap Y_{2} \subseteq \operatorname{Soc}_{s}\left(M_{1} \oplus Y_{2}\right)$. As a result $M$ is $s s$-lifting.

\section{REFERENCES}

[1] E. Büyükaş1k and Y. M. Demirci, "Weakly distributive modules. Applications to supplement submodules." Proc. Indian Acad. Sci., Math. Sci., vol. 120, no. 5, pp. 525-534, 2010, doi: 10.1007/s12044-010-0053-9.

[2] A. Ç. Özcan, A. Harmanci, and P. F. Smith, "Duo modules." Glasg. Math. J., vol. 48, no. 3, pp. 533-545, 2006, doi: 10.1017/S0017089506003260.

[3] N. V. John Clark, Christian Lomp and R. Wisbauer, Lifting Modules: Supplements and Projectivity in Module Theory. Basel: Birkhäuser, 2006. doi: 10.1007/3-7643-7573-6.

[4] E. Kaynar, E. Türkmen, and H. Çalışıcı, "Ss-supplemented modules," Communications Faculty of Sciences University of Ankara Series A1 Mathematics and Statistics, vol. 69, pp. 473 - 485, 2020, doi: 10.31801/cfsuasmas.585727.

[5] D. Keskin, "Finite direct sums of (d1)-modules," Turkish Journal of Mathematics, vol. 22, pp. 85 92, 1998.

[6] S. H. Mohamed and B. J. Müller, Continuous and discrete modules. Cambridge etc.: Cambridge University Press, 1990, vol. 147, doi: 10.1017/CBO9780511600692.

[7] R. Wisbauer, Foundations of module and ring theory. A handbook for study and research. Revised and updated Engl. ed., revised and updated engl. ed. ed. Philadelphia etc.: Gordon and Breach Science Publishers, 1991, vol. 3, doi: 10.1201/9780203755532.

[8] D. X. Zhou and X. R. Zhang, "Small-essential submodules and Morita duality." Southeast Asian Bull. Math., vol. 35, no. 6, pp. 1051-1062, 2011.

Author's address

Figen Eryilmaz

Ondokuz Mayis University, Department of Mathematics Education, Kurupelit, Atakum, 55139 Samsun, Turkey

E-mail address: fyuzbasi@omu.edu.tr 